\title{
AUTOIMMUNE MENINGOENCEPHALITIS INDUCED BY IMMUNE-CHECKPOINT INHIBITOR USE: A SINGLE CENTER EXPERIENCE
}

\author{
Shin Hye Yoo', Miso Kim ${ }^{1}$, Soon-Tae Lee ${ }^{2}$, Bhumsuk Keam ${ }^{1}$
}

SNUH

${ }^{1}$ Department of Internal Medicine, Seoul National University Hospital, Seoul, Korea;

${ }^{2}$ Department of Neurology, Seoul National University Hospital, Seoul, Korea;

\section{INTRODUCTION}

- Immune checkpoint inhibitors (ICls) have emerged as promising class of anti-cancer treatment in several types of tumor, but immune-related adverse events are occasionally encountered during $\mathrm{ICl}$ treatment.

- Autoimmune meningoencephalitis is a rare but fatal adverse event. However, little has been known about the natural course and optimal strategies to manage immune-related meningoencephalitis (irME).

\section{METHODS}

Retrospective review of clinical characteristics and the natural course of three urothelial carcinoma of bladder patients who developed irME induced by $\mathrm{ICl}$

\section{RESULTS}

\section{Presenting symptoms}

- The most common presenting symptoms were fever and altered mentality, with the median onset of 14 days.

Measurements

- Cerebrospinal fluid analyses revealed pleocytosis and elevated protein level, but negative results for infectious etiologies.

- Malignant cells were not detected in cerebrospinal fluid.

- Paraneoplastic antibody was observed in one patient (Case 1).

Treatments

- As irME was highly suspected, the patients were given steroid.

- Case 1 was completely recovered with concurrent steroid plus immunoglobulin (IVIG).

- Case 2 received IVIG as he did not show improvement with steroid and responded transiently, but finally recurred.

- In case 3, steroid resolved her altered mentation, but weakness of bilateral extremities due to Guillain-Barré syndrome remained and was partially recovered with IVIG and intravenous rituximab.

Tumor response

- Tumor size was decreased in 2 patients (Case 2, Case 3).

\begin{tabular}{|c|c|c|c|c|c|c|c|c|c|}
\hline No. & $\begin{array}{l}\text { Age } \\
\text { /Sex }\end{array}$ & Drug & Disease & $\begin{array}{l}\text { PD-L1 expre } \\
\text { ssion by IHC } \\
\text { (SP142*) }\end{array}$ & $\begin{array}{c}\text { Time to } \\
\text { onset }\end{array}$ & 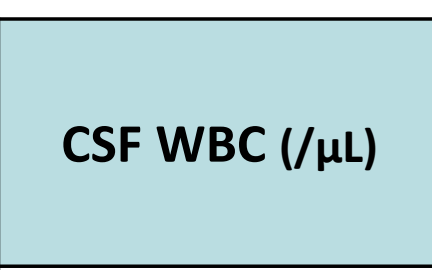 & $\begin{array}{c}\text { CSF } \\
\text { protein } \\
(\mathrm{mg} / \mathrm{dL}) \\
\end{array}$ & $\begin{array}{l}\text { Immunosuppressive Treatment } \\
\text { (D: day from onset) }\end{array}$ & $\begin{array}{l}\text { Time to } \\
\text { recovery }\end{array}$ \\
\hline 1 & $42 / M$ & $\begin{array}{l}\text { Anti-PD-L1 antibody } \\
\text { plus MEK inhibitor }\end{array}$ & $\begin{array}{l}\text { Urothelial cell car } \\
\text { cinoma of bladder } \\
\text { (HER2-amplified) }\end{array}$ & Negative & 14 days & 65 (P O L 60 58) & 133 & $\begin{array}{l}\text { Steroid (mPd 1g daily } 5 \text { days) (D3-7) } \\
\text { IVIG (0.4 g/kg/day } 5 \text { days) (D3-7) }\end{array}$ & 10 days \\
\hline 2 & $49 / M$ & $\begin{array}{l}\text { atezolizumab } \\
\left(\text { TECENTRIQ }^{\circledR}\right)\end{array}$ & $\begin{array}{l}\text { Urothelial cell car } \\
\text { cinoma of bladder } \\
\text { (HER2-amplified) }\end{array}$ & $\begin{array}{l}\text { Positive } \\
1 \% \text { on } \mathrm{TC} \\
7 \% \text { on IC }\end{array}$ & 14 days & 50 (P 17 L 7 O 76) & 218.1 & $\begin{array}{c}\text { Steroid (mPd 1g daily } 5 \text { days) (D3-7) } \\
\text { IVIG (0.4 g/kg/day } 5 \text { days) (D5-9; D4 } \\
\text { 4-48) }\end{array}$ & Death \\
\hline 3 & $70 / F$ & $\begin{array}{l}\text { atezolizumab } \\
\left(\text { TECENTRIQ }^{\circledR}\right)\end{array}$ & $\begin{array}{l}\text { Urothelial cell car } \\
\text { cinoma of bladder }\end{array}$ & $\begin{array}{l}\text { Positive } \\
1 \% \text { on TC } \\
5 \% \text { on IC }\end{array}$ & 15 days & 30 (P 6 L 60 18) & 358 & $\begin{array}{l}\text { Steroid (mPd } 1 \mathrm{mg} / \mathrm{kg} \text { daily, then ta } \\
\text { pering to } 15 \mathrm{mg} \text { daily) (D4-) } \\
\text { IVIG (0.4 } \mathrm{g} / \mathrm{kg} / \text { day } 5 \text { days) (D6-10; D } \\
\text { 27-31; D52-56) } \\
\text { Mycophenolate mofetil (500mg tw } \\
\text { ice a day) (D49-) } \\
\text { Rituximab ( } 375 \mathrm{mg} / \mathrm{m} 2 \mathrm{IV}) \text { (D47, D7 } \\
1, \mathrm{D} 78, \mathrm{D} 88)\end{array}$ & $\begin{array}{l}22 \text { days } \\
80 \text { days } \\
\text { (to partial } \\
\text { recovery o } \\
\text { f GBS) }\end{array}$ \\
\hline
\end{tabular}

Abbreviation: M, male; F, female; PD-L1, programmed death-ligand 1; MEK, mitogen-activated protein kinase kinase; HER-2, human epidermal growth factor receptor-2; IHC, immunohistochemistry; TC, tumor cell; IC, immune cell; CSF, cerebrospinal fluid; WBC, white blood count; P, polymorphonuclear cell; L, lymphocyte; O, other cells; mPd, methylprednisolone; IVIG, intravenous immunoglobulin; GBS, Guillain-Barré syndrome;

* VENTANA PD-L1 (SP142) Assay (Ventana Medical Systems Inc., Tucson, AZ)

\section{CONCLUSIONS}

irME associated with ICl develops early after the first dose given within two weeks. The natural course varies and fatal. The prompt use of high dose steroid and IVIG treatment after exclusion of infectious cause might be essential for neurologic recovery.

Keywords: immune checkpoint inhibitor; meningoencephalitis; neurological adverse event; steroid; intravenous immunoglobulin; 